\title{
Red Cell Distribution Width and Mortality in Patients With Acute Coronary Syndrome: A Meta-Analysis on Prognosis
}

\author{
Lauro L. Abrahan, IV a , John Daniel A. Ramos ${ }^{\mathrm{a}}$, Elleen L. Cunanan ${ }^{\mathrm{a}}$, \\ Marc Denver A. Tiongson a, b, Felix Eduardo R. Punzalan ${ }^{\mathrm{a}}$
}

\begin{abstract}
Background: Red cell distribution width (RDW), a routine component of the complete blood count (CBC), measures variation in the size of circulating erythrocytes. It has been associated with several clinical outcomes in cardiovascular disease. We sought to strengthen the association between RDW and mortality in patients admitted for acute coronary syndrome (ACS) by pooling together data from available studies.

Methods: Studies that fulfilled the following were identified for analysis: 1) observational; 2) included patients admitted for ACS; 3) reported data on all-cause or cardiovascular (CV) mortality in association with a low or high RDW; and 4) used logistic regression analysis to control for confounders. Using MEDLINE, Clinical Key, ScienceDirect, Scopus, and Cochrane Central Register of Controlled Trials databases, a search for eligible studies was conducted until January 9, 2017. The quality of each study was evaluated using the Newcastle-Ottawa Quality Assessment Scale. Our primary outcome of interest was all-cause or CV mortality. We also investigated the impact of RDW on major adverse cardiovascular events (MACEs) for the studies that reported these outcomes. Review Manager (RevMan) 5.3 was utilized to perform Mantel-Haenzel analysis of random effects and compute for relative risk.
\end{abstract}

Results: We identified 13 trials involving 10,410 patients, showing that in ACS, a low RDW is associated with a statistically significant lower all-cause or CV mortality (RR 0.35 , (95\% CI 0.30 to 0.40$)$, P $<0.00001, \mathrm{I}^{2}=53 \%$ ), a finding that was consistent both in the shortand long-term.

Conclusions: A low RDW is also associated with lower risk for MACEs after an ACS (RR 0.56, (95\% CI 0.51 to 0.61$), \mathrm{P}<0.00001$, $\left.\mathrm{I}^{2}=91 \%\right)$. A low RDW during an ACS is associated with lower allcause or CV mortality and lower risk of subsequent MACEs, providing us with a convenient and inexpensive risk stratification tool in

Manuscript submitted May 15, 2018, accepted May 22, 2018

aSection of Cardiology, University of the Philippines, Philippine General Hospital, Manila Philippines 1000, Philippines

${ }^{b}$ Corresponding Author: Marc Denver A. Tiongson, Section of Cardiology, University of the Philippines, Philippine General Hospital, Manila Philippines 1000, Philippines. Email: denver_tiongson@yahoo.com

doi: https://doi.org/10.14740/cr732w
ACS patients.

Keywords: Red cell distribution width; Acute coronary syndrome; Myocardial infarction

\section{Introduction}

The red cell distribution width (RDW), a routinely reported parameter in the complete blood count of most laboratories, is a numerical measure of the variability in size of circulating erythrocytes [1]. A higher RDW value indicates greater variation in size, and is the objective equivalent of anisocytosis noted in peripheral blood smears [2]. It has found utility in the work-up for the differential diagnoses of microcytic anemia [3-4].

The past decade has seen a rise in studies focusing on the use of RDW as a novel marker of prognosis and severity of several cardiovascular diseases. For example, Isik et al [5] demonstrated that a high RDW was an independent predictor not only of angiographically documented coronary artery disease (CAD), but also of more complex coronary artery lesions, as reflected by higher SYNTAX (Synergy between PCI with Taxus and Cardiac Surgery) scores. Research examining other diseases attributed to atherosclerosis, such as ischemic stroke and peripheral arterial disease (PAD), yielded similar findings. Jia et al [6] showed that elevated RDW was significantly and independently associated with carotid intimal medial thickness (IMT, a surrogate measure of progression of carotid atherosclerosis), and this was correlated clinically in a study by Kim et al [7], where they found that RDW was also associated with poor functional outcome and all-cause mortality at 3 months post-ictus. For patients with PAD, RDW was seen to increase gradually across Fontaine classification stages in a paper by Demirtas et al [8], while Ye et al [9] noted that a $1 \%$ incremental increase in RDW was associated with a $10 \%$ greater risk of all-cause mortality.

In terms of atrial fibrillation (AF), RDW was observed to be an independent predictor for the development of nonvalvular AF [10]. It also had predictive value for post-operative $\mathrm{AF}$ in patients undergoing elective on-pump cardiac surgeries [11, 12], as well as the risk for both all-cause mortality and major adverse cardiovascular events (MACEs) in patients already with AF at baseline [13]. Beginning with Felker's 
landmark study [14], there have been an extensive number of studies published regarding the significance of RDW in heart failure (HF) patients, and a meta-analysis by Huang et al [15] concluded that increased RDW, whether at baseline or upon discharge, was associated with poor HF prognosis.

Since prognosis ultimately affects the urgency and aggressiveness of acute coronary syndrome (ACS) management, our group wished to focus on this particular subset of CAD patients. We therefore synthesized the available evidence involving patients with ACS to determine the association between RDW and mortality for this specific population. Our objectives are: 1) To determine the association of RDW with mortality in patients with ACS; 2) To determine the association of RDW with MACEs in patients with ACS.

\section{Methods}

\section{Literature search}

The four authors of this review independently searched the MEDLINE, Clinical Key, ScienceDirect, Scopus, and Cochrane Central Register of Controlled Trials databases for eligible studies published up to January 9, 2017. The search terms used were "red cell distribution width," "RDW," "acute coronary syndrome," and "myocardial infarction." In addition, we also examined the reference lists of primary studies and review articles, as well as links to related or similar papers. Only human studies and articles with full text in English were included.

\section{Inclusion and exclusion criteria}

Studies that fulfilled the following inclusion criteria were identified for analysis: 1) observational study design; 2) included patients admitted primarily for ACS; 3 ) reported data on allcause or cardiovascular (CV) mortality in association with a low or high RDW; and 4) used logistic regression analysis to control for confounders. Studies involving patients with stable angina or stable CAD were excluded.

\section{Data extraction}

The Newcastle-Ottawa Scale (NOS) [16] was used to assess the quality of studies included for analysis. Using the NOS tool, a study may be awarded a maximum of nine stars to indicate high quality, and it makes use of three major domains, namely selection of the study groups, comparability of the two groups, and assessment of outcome. Two of the authors (LL Abrahan IV and EL Cunanan) independently performed quality assessment, and differences were resolved by consultation with the third author (either JDA Ramos or MDA Tiongson) who was blinded to the previous assessment.

For the included studies, the data extracted were the following: author/s, year published, study design, population, sample size, outcome events (all-cause or cardiovascular mortality, MACEs), and follow-up duration. The protocol for data extraction was similar to that for quality assessment, with disputes once again resolved by discussion with the third author.

\section{Statistical analysis}

Meta-analysis was conducted using the Mantel-Haenzel random-effects model to generate risk ratios, 95\% confidence intervals (CIs), and forest plots. Heterogeneity was assessed through the $\mathrm{I}^{2}$ test, and prespecified subgroup and sensitivity analyses were performed. For subgroup analysis, the included studies were categorized according to duration of follow-up $(<1$ year vs. $\geq 1$ year) and study design (prospective vs. retrospective).

Sensitivity analysis was done to evaluate the stability of pooled estimates and check for any significant change in results after individually excluding each of the studies. A study would be considered an outlier if its exclusion from the analysis produced a 95\% confidence interval that did not overlap with the $95 \%$ confidence interval of the original pooled estimate that included all studies. Funnel plot analysis was used to evaluate for publication bias. All statistical analyses were carried out using Review Manager (RevMan) version 5.3 (The Nordic Cochrane Centre, The Cochrane Collaboration, Copenhagen).

\section{Results}

\section{Literature search}

Our systematic literature search yielded an initial total of 167 potential articles. Figure 1 shows the flow chart for the identification process, and the pool was subsequently narrowed down to 13 studies involving 10,410 ACS patients for inclusion in the meta-analysis.

\section{Study characteristics}

Table 1 [17-29] summarizes the details of the included studies. These studies were published from 2010 to 2016, with population sizes ranging from 100 to 2,506. Six of the studies had a prospective study design, while the rest were retrospective in nature. Study duration varied from 1 month to 4 years, with one study (Ilhan et al [23]) notably limited to in-hospital (prior to discharge) mortality outcome. The study populations were composed of varying permutations of ACS phenotypes (ST elevation myocardial infarction or STEMI, non-ST elevation myocardial infarction or NSTEMI, and unstable angina or UA). For the eight studies that also examined MACEs, these events had several definitions including, but not limited to: reinfarction, the need for repeat revascularization, cardiogenic shock, heart failure, cardiac dysrhythmias, and stroke.

\section{Quality assessment}

For quality assessment, six of the included studies were given 


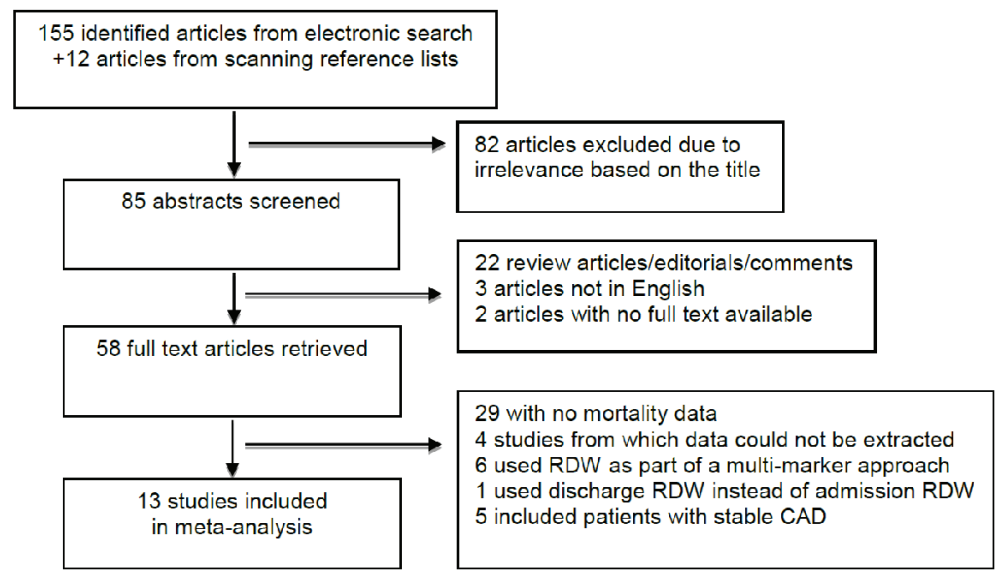

Figure 1. Flow diagram for study identification and inclusion.

the highest rating of nine stars on the Newcastle-Ottawa Scale, with the rest of the studies receiving seven to eight stars (Table 2 [17-29]). The study by Cavusoglu et al [19] had a submaximal score for cohort selection because it only included male patients. Six of the studies did not control for echocardiographic left ventricular ejection fraction during multivariate analysis. Two studies were given submaximal scores for outcome assessment due to very short follow-up duration (1 month or less).

\section{RDW and mortality in ACS patients}

The pooled risk ratio from the combined data of the 13 trials (Fig. 2) showed that a low RDW value upon admission of patients with ACS is associated with significantly lower all-cause or cardiovascular mortality $(6.0 \%$ for low RDW vs. $16.3 \%$ for high RDW) (RR 0.35, (95\% CI 0.30 to 0.40$), \mathrm{P}<0.00001)$. There was a moderate degree of heterogeneity, with an $\mathrm{I}^{2}$ value of $53 \%$. On sensitivity analysis, the overall results remained robust, and no studies were removed as statistical outliers. To further investigate for possible sources of heterogeneity, we proceeded with subgroup analysis.

We first classified the included studies according to the duration of follow-up $(<1$ year or short-term vs. $\geq 1$ year or long-term, Fig. 3). Both subgroups still showed a consistent association of low RDW with lower risk of death. However, compared to the primary analysis that included all 13 studies, the subgroup with shorter follow-up yielded a lower pooled RR of 0.23 (95\% CI 0.15 to $0.34, \mathrm{P}<0.00001)$, while the subgroup with longer follow-up resulted in a slightly higher pooled RR of 0.39 (95\% CI 0.32 to $0.47, \mathrm{P}<0.00001)$. The $\mathrm{I}^{2}$ values for both short and long-term subgroups $(23 \%$ and $25 \%$, respectively) were lower than the $\mathrm{I}^{2}$ value of the original analysis (53\%). The test for subgroup differences resulted in a high $\mathrm{I}^{2}$ value of $81.6 \%$.

We then categorized the studies according to study design (prospective vs. retrospective, Fig. 4). Pooled RR estimates remained similar to that of the original analysis (RR $0.30(95 \%$ CI 0.19 to 0.46$), \mathrm{P}<0.00001$ for prospective; RR $0.32(95 \%$
CI 0.24 to 0.42$), \mathrm{P}<0.00001$ for retrospective)]. $\mathrm{I}^{2}$ values within the prospective and retrospective subgroups were $66 \%$ and $41 \%$, respectively. The test for subgroup differences yielded an $\mathrm{I}^{2}$ value of $0 \%$

Funnel plot analysis for the 13 studies (Fig. 5) showed a relatively symmetric plot with most points clustered in the midline, suggesting the absence of significant publication bias.

\section{RDW and MACEs in ACS patients}

For the eight studies that recorded MACEs as outcomes (Fig. 6), the pooled RR demonstrated a low admission RDW was also associated with lower risk for MACEs after an ACS (23.3\% for low RDW vs. 32.9\% for high RDW) (RR 0.56, (95\% CI 0.51 to 0.61$), \mathrm{P}<0.00001)$. The heterogeneity was considerably high for this analysis, with a value of $\mathrm{I}^{2}=91 \%$.

\section{Discussion}

The present systematic review and meta-analysis indicates that a low RDW on admission in patients suffering from ACS is associated with a lower risk of both death and subsequent MACEs. Previous meta-analyses have studied the association between RDW and mortality in patients with CAD [30] and with heart failure [15], but to our knowledge, our study is the first meta-analysis to examine RDW on the background of ACS as a specific subpopulation.

We have shown that the risk for cardiovascular or allcause death for ACS patients with a low admission RDW is only approximately one third of the risk for patients with a high RDW (RR 0.35, (95\% CI 0.30 to 0.40$)$ ). There was, however, a moderate degree of heterogeneity $\left(\mathrm{I}^{2}=53 \%\right)$ among the 13 studies in this primary analysis. Sensitivity analysis showed that there were no particular outliers that skewed the data in a specific direction. On subgroup analysis, the duration of follow-up (with a cut-off of 1 year) appeared to be a significant source of heterogeneity, since the specific grouping of studies drove down the $\mathrm{I}^{2}$ values within each subgroup. This 
Table 1. Characteristics of Studies Included in Meta-Analysis

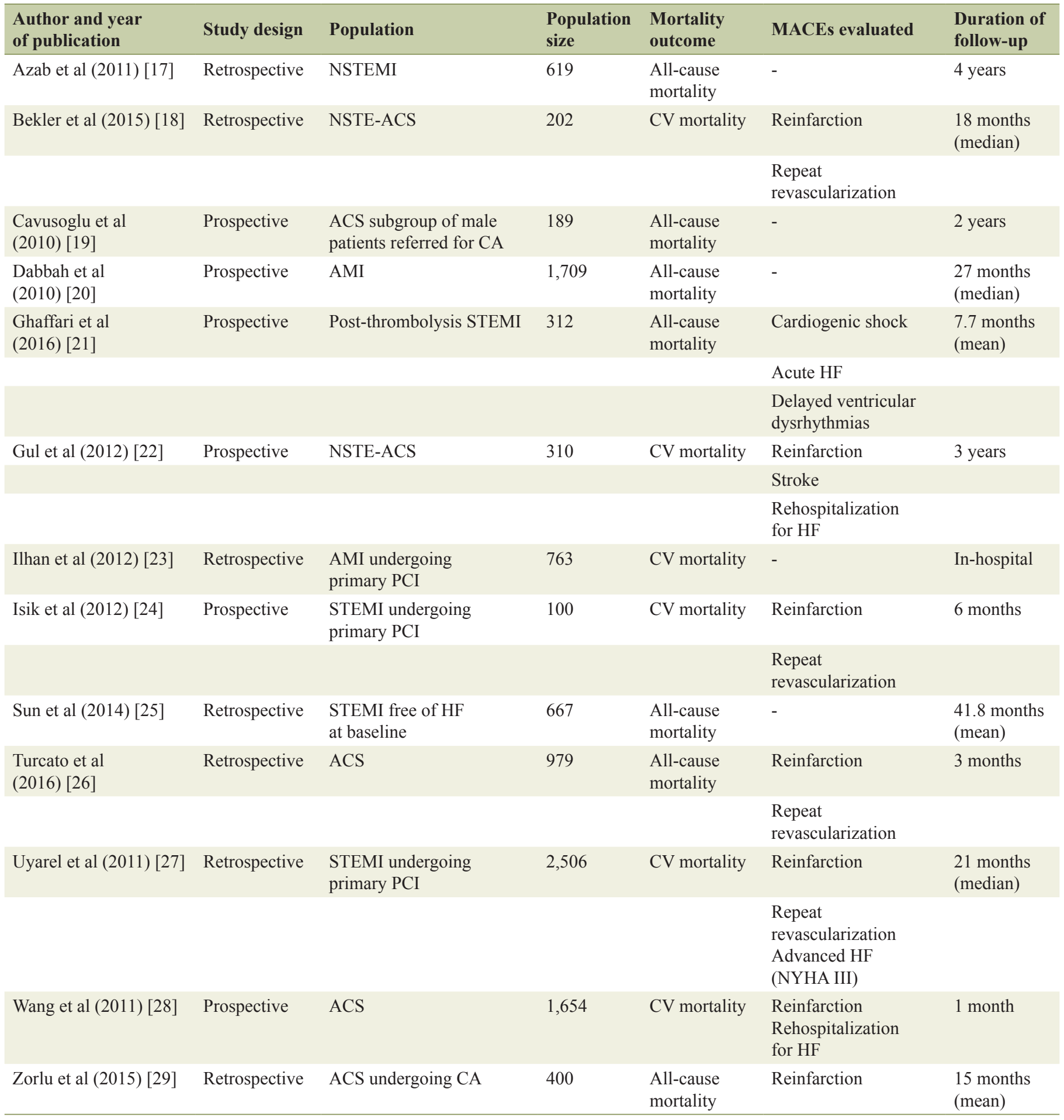

MACE: major adverse cardiovascular event; CAD: coronary artery disease; ACS: acute coronary syndrome; NSTE-ACS: non-ST elevation acute coronary syndrome; AMI: acute myocardial infarction; STEMI: ST elevation myocardial infarction; PCI: percutaneous coronary intervention; CA: coronary angiography; CV: cardiovascular; HF: heart failure; NYHA: New York Heart Association.

was further confirmed by the test of subgroup differences $\left(\mathrm{I}^{2}\right.$ $=81.6 \%$ ). Expectedly, the subgroup with the longer followup ( $\geq 1$ year) had the seemingly higher risk ratio $(0.39$ for long-term vs. 0.23 for short-term), likely since a longer time period would allow for more events to occur. This implies that shorter follow-up tends to underestimate the prognostic value 
Table 2. Quality Assessment of Included Studies Using Newcastle-Ottawa Scale

\begin{tabular}{|c|c|c|c|c|}
\hline Author and year of publication & Selection & Comparability & Outcome & Total rating \\
\hline Azab et al (2011) [17] & $\star \star \star \star$ & $\star \star$ & $\star \star \star$ & $9 \star$ \\
\hline Bekler et al (2015) [18] & $\star \star \star \star ~$ & $\star \star$ & $\star \star \star$ & $9 \star$ \\
\hline Cavusoglu et al (2010) [19] & $\star \star \star$ & $\star$ & $\star \star \star$ & $7 \star$ \\
\hline Dabbah et al (2010) [20] & $\star \star \star \star$ & $\star \star$ & $\star \star \star$ & $9 \star$ \\
\hline Ghaffari et al (2016) [21] & $\star \star \star \star$ & $\star \star$ & $\star \star \star$ & $9 \star$ \\
\hline Gul et al (2012) [22] & $\star \star \star \star$ & $\star \star$ & $\star \star \star$ & $9 \star$ \\
\hline Ilhan et al (2012) [23] & $\star \star \star \star$ & $\star$ & $\star \star$ & $7 \star$ \\
\hline Isik et al (2012) [24] & $\star \star \star \star ~$ & $\star$ & $\star \star \star$ & $8 \star$ \\
\hline Sun et al (2014) [25] & $\star \star \star \star$ & $\star$ & $\star \star \star$ & $8 \star$ \\
\hline Turcato et al (2016) [26] & $\star \star \star \star ~$ & $\star$ & $\star \star \star$ & $8 \star$ \\
\hline Uyarel et al (2011) [27] & $\star \star \star \star$ & $\star$ & $\star \star \star$ & $8 \star$ \\
\hline Wang et al (2011) [28] & $\star \star \star \star$ & $\star \star$ & $\star \star$ & $8 \star$ \\
\hline Zorlu et al (2015) [29] & $\star \star \star \star$ & $\star \star$ & $\star \star \star$ & $9 \star$ \\
\hline
\end{tabular}

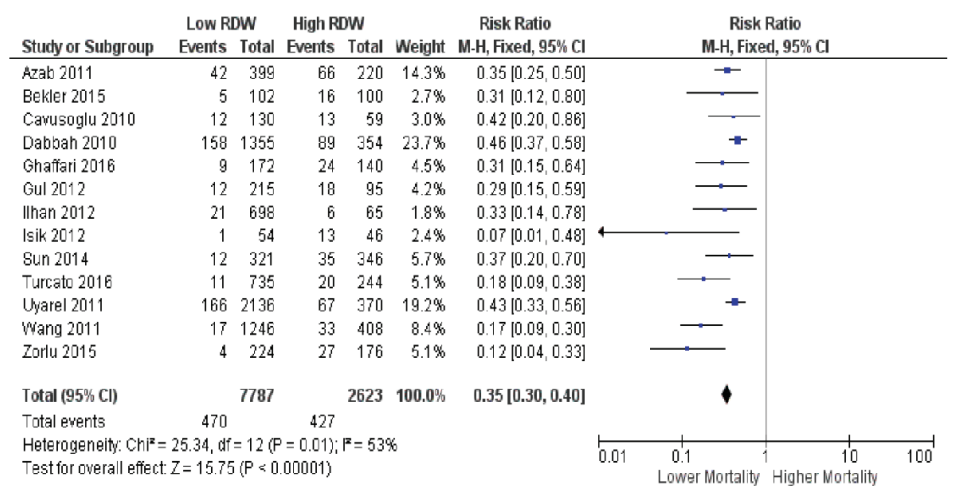

Figure 2. Forest plot showing pooled risk ratio of RDW and all-cause or cardiovascular mortality.

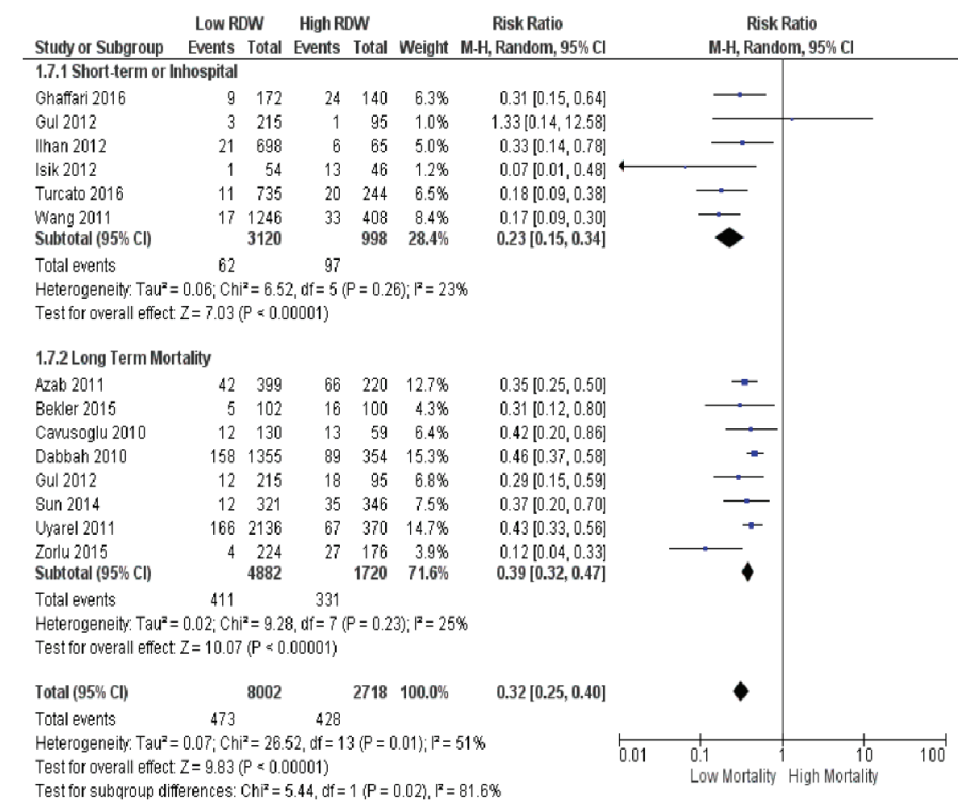

Figure 3. Subgroup analysis according to duration of follow-up (<1 year vs. $\geq 1$ year). 


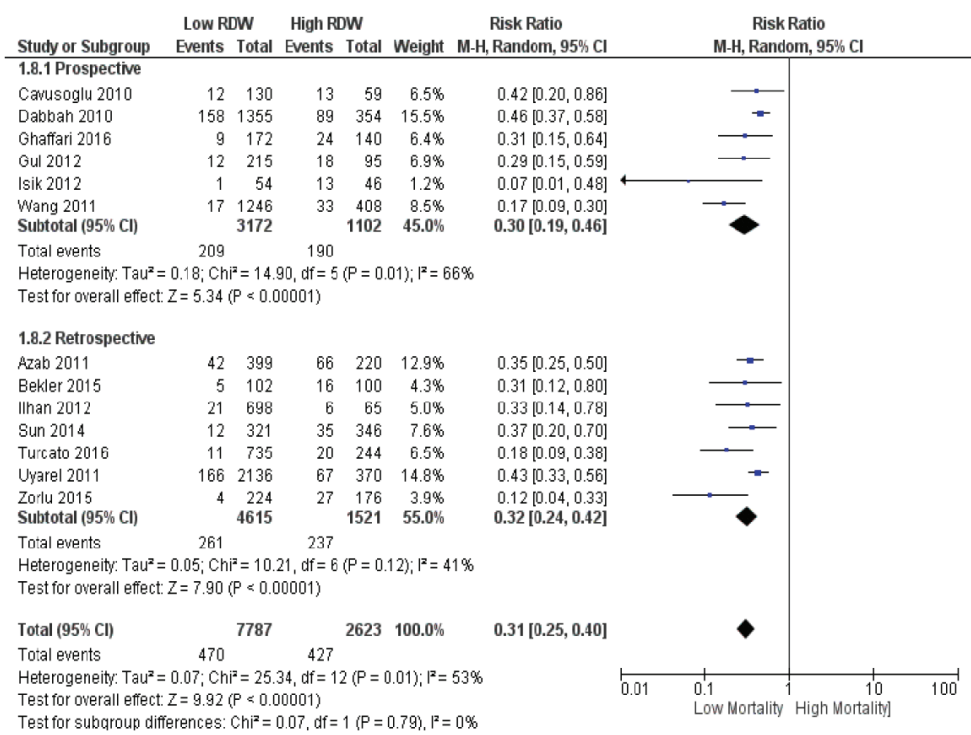

Figure 4. Subgroup analysis according to study design (prospective vs. retrospective).

of RDW.

At the outset, we hypothesized that study design (prospective vs. retrospective) would also be a significant source of heterogeneity. Subgrouping by study design, however, did not seem to have an apparent effect on the pooled RRs for the subgroups. Heterogeneity remained within the subgroups $\left(\mathrm{I}^{2}=66 \%\right.$ for prospective; $\mathrm{I}^{2}=41 \%$ for retrospective), and the test for subgroup differences revealed homogeneity $\left(\mathrm{I}^{2}=\right.$ $0 \%$ ). Therefore, study design was not a significant source of heterogeneity for this analysis. Other factors that we identified as possible sources of heterogeneity include variations in study population (STEMI vs. NSTE-ACS vs. both), primary outcomes of interest, definitions of outcomes (especially for the MACEs), approaches in defining RDW categories (dichotomous vs. tertiles or quartiles), and the specific RDW assays that were utilized.
For the analysis of MACEs, the results showed that the risk for MACEs following an ACS in patients with a low admission RDW is only around half that of patients with a high RDW (RR 0.56, (95\% CI 0.51 to 0.61$)$ ). In this analysis, heterogeneity was predictably high $\left(\mathrm{I}^{2}=91 \%\right)$, given the different outcomes that were considered as MACEs in the individual studies, as well as the varying definitions for each particular outcome.

A particular strength of our study is the only moderate degree of heterogeneity, considering that the included studies are all observational in nature rather than randomized controlled trials. This heterogeneity was further minimized with subgroup analysis, pinpointing follow-up duration as one of the major contributors. In addition, the overall results were robust on sensitivity analysis. Included studies were of at least moderate quality, and there was no evidence of publication bias on

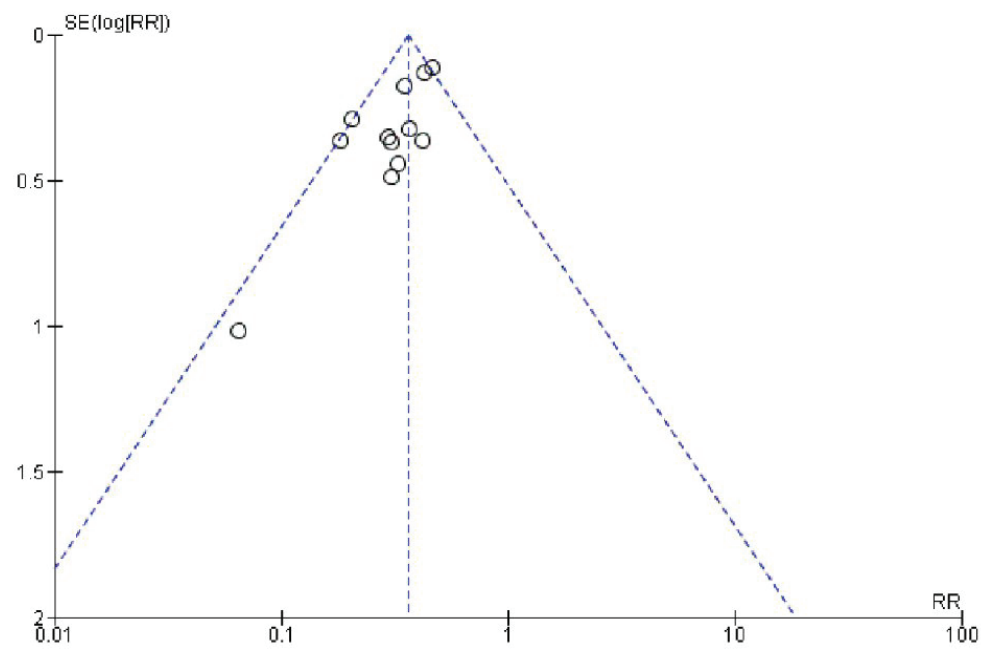

Figure 5. Funnel plot for RDW and all-cause or cardiovascular mortality. 


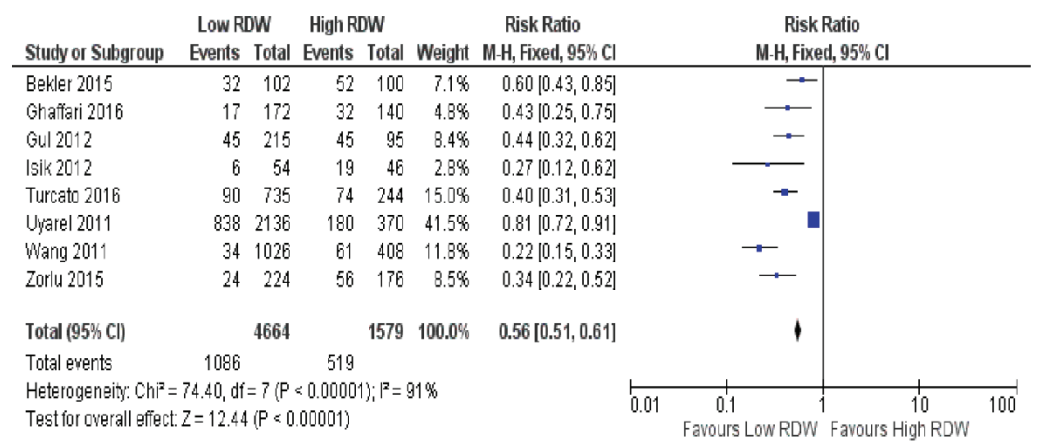

Figure 6. Forest plot showing pooled risk ratio of RDW and major adverse cardiovascular events (MACEs).

funnel plot analysis.

Still, this analysis was not without its limitations. We had to exclude three articles since they were not written in English, opening up the possibility of study selection bias. Furthermore, although we selected studies that controlled for most of the important confounders that affect mortality in ACS patients (such as age, cardiovascular comorbidities, Killip classification, and LV ejection fraction), we did not screen for control of confounders for RDW. These include hematological factors such as anemia and folate or vitamin B12 deficiency.

The underlying atherosclerotic process can be considered the pathogenetic link that explains why there is a high variability in erythrocyte volume in ACS and other related cardiovascular conditions. The inflammatory state in atherosclerosis [31] leads to ineffective erythropoiesis and release of larger, immature erythrocytes from bone marrow $[32,33]$, which then skews the RDW towards higher values. Delayed RBC clearance which is also associated with pathology and all-cause mortality has been proposed as another possible mechanism of increased RDW. Since RBCs normally grow smaller in size throughout their lifespan, postponing RBC elimination allows smaller erythrocytes to stay in the circulation, thus accounting for the low-volume tail-end of red cell volume distribution [34]. In addition, impaired iron mobilization may also play a contributing factor [32].

With the strength of evidence presented in this analysis, we therefore propose the use of RDW as a risk stratification tool for ACS patients. Knowing the prognosis of a patient in terms of the risk for death and MACEs can guide key points in clinical decision-making, such as the urgency for invasive angiography and possible revascularization. RDW is a simple and inexpensive diagnostic test, and is readily available as an automatic component of complete blood counts of laboratories worldwide. Also, since the lifespan of erythrocytes extends up to 120 days, RDW is less susceptible to biological variations over time compared to other conventional serum markers of ACS such as cardiac troponins.

We cannot ascertain from the results of this study whether RDW is a causative factor for mortality or MACEs in ACS patients. To determine this, large controlled trials to test RDWmodifying therapies would have to be performed. We would first recommend validating the utility of RDW as a prognostic marker in a Filipino ACS cohort through our own local stud- ies. From there, we can explore the possibility of incorporating RDW into risk scoring systems or providing incremental prognostic value by combining it with other serum inflammatory markers.

\section{Conclusions}

Our study has demonstrated that RDW is associated with risk for mortality and MACEs in patients diagnosed with ACS. Relatively affordable, convenient, and readily available, RDW can be used as a risk stratification tool in our resource-limited setting.

\section{References}

1. Arbel Y, Birati EY, Finkelstein A, Halkin A, Berliner S, Katz BZ, Revivo M, et al. Red blood cell distribution width and 3-year outcome in patients undergoing cardiac catheterization. J Thromb Thrombolysis. 2014;37(4):469474.

2. Aulakh R, Sohi I, Singh T, Kakkar N. Red cell distribution width (RDW) in the diagnosis of iron deficiency with microcytic hypochromic anemia. Indian J Pediatr. 2009;76(3):265-268.

3. Viswanath D, Hegde R, Murthy V, Nagashree S, Shah R. Red cell distribution width in the diagnosis of iron deficiency anemia. Indian J Pediatr. 2001;68(12):1117-1119.

4. Buch AC, Karve PP, Panicker NK, Singru SA, Gupta $\mathrm{SC}$. Role of red cell distribution width in classifying microcytic hypochromic anaemia. J Indian Med Assoc. 2011;109(5):297-299.

5. Isik T, Uyarel H, Tanboga IH, Kurt M, Ekinci M, Kaya A, Ayhan E, et al. Relation of red cell distribution width with the presence, severity, and complexity of coronary artery disease. Coron Artery Dis. 2012;23(1):51-56.

6. Jia H, Li H, Zhang Y, Li C, Hu Y, Xia C. Association between red blood cell distribution width (RDW) and carotid artery atherosclerosis (CAS) in patients with primary ischemic stroke. Arch Gerontol Geriatr. 2015;61(1):7275.

7. Kim J, Kim YD, Song TJ, Park JH, Lee HS, Nam CM, 
Nam HS, et al. Red blood cell distribution width is associated with poor clinical outcome in acute cerebral infarction. Thromb Haemost. 2012;108(2):349-356.

8. Demirtas S, Karahan O, Yazici S, Guclu O, Caliskan A, Yavuz C, Kucuker A, et al. The relationship between complete blood count parameters and Fontaine's Stages in patients with peripheral arterial disease. Vascular. 2014;22(6):427-431.

9. Ye Z, Smith C, Kullo IJ. Usefulness of red cell distribution width to predict mortality in patients with peripheral artery disease. Am J Cardiol. 2011;107(8):12411245.

10. Gungor B, Ozcan KS, Erdinler I, Ekmekci A, Alper AT, Osmonov D, Calik N, et al. Elevated levels of RDW is associated with non-valvular atrial fibrillation. J Thromb Thrombolysis. 2014;37(4):404-410.

11. Ertas G, Aydin C, Sonmez O, Erdogan E, Turfan M, Tasal A, Bacaksiz A, et al. Red cell distribution width predicts new-onset atrial fibrillation after coronary artery bypass grafting. Scand Cardiovasc J. 2013;47(3):132-135.

12. Korantzopoulos P, Liu T. RDW as a marker of postoperative atrial fibrillation. Int J Cardiol. 2015;191:109.

13. Wan H, Yang Y, Zhu J, Huang B, Wang J, Wu S, Shao $\mathrm{X}$, et al. The relationship between elevated red cell distribution width and long-term outcomes among patients with atrial fibrillation. Clin Biochem. 2015;48(12):762767.

14. Felker GM, Allen LA, Pocock SJ, Shaw LK, McMurray JJ, Pfeffer MA, Swedberg K, et al. Red cell distribution width as a novel prognostic marker in heart failure: data from the CHARM Program and the Duke Databank. J Am Coll Cardiol. 2007;50(1):40-47.

15. Huang YL, Hu ZD, Liu SJ, Sun Y, Qin Q, Qin BD, Zhang WW, et al. Prognostic value of red blood cell distribution width for patients with heart failure: a systematic review and meta-analysis of cohort studies. PLoS One. 2014;9(8):e104861.

16. Wells GA, Shea B, O'Connell D, Peterson J, Welch V, Losos M, Tugwell P. The Newcastle-Ottawa Scale (NOS) for assessing the quality of nonrandomised studies in meta-analyses. 2011. Available: http://www.ohri.ca/programs/clinical_epidemiology/oxford.asp. Accessed January $9,2017$.

17. Azab B, Torbey E, Hatoum H, Singh J, Khoueiry G, Bachir R, McGinn JT, Jr., et al. Usefulness of red cell distribution width in predicting all-cause long-term mortality after non-ST-elevation myocardial infarction. Cardiology. 2011;119(2):72-80.

18. Bekler A, Tenekecioglu E, Erbag G, Temiz A, Altun B, Barutcu A, Gazi E, et al. Relationship between red cell distribution width and long-term mortality in patients with non-ST elevation acute coronary syndrome. Anatol J Cardiol. 2015;15(8):634-639.

19. Cavusoglu E, Chopra V, Gupta A, Battala VR, Poludasu S, Eng C, Marmur JD. Relation between red blood cell distribution width (RDW) and all-cause mortality at two years in an unselected population referred for coronary angiography. Int J Cardiol. 2010;141(2):141-146.

20. Dabbah S, Hammerman H, Markiewicz W, Aronson D.
Relation between red cell distribution width and clinical outcomes after acute myocardial infarction. Am J Cardiol. 2010;105(3):312-317.

21. Ghaffari S, Pourafkari L, Sepehrvand N, Aslanabadi N, Faridi L, Tajlil A, Masoumi N, et al. Red cell distribution width is a predictor of ST resolution and clinical outcome following thrombolysis in acute ST elevation myocardial infarction. Thromb Res. 2016;140:1-6.

22. Gul M, Uyarel H, Ergelen M, Karacimen D, Ugur M, Turer A, Bozbay M, et al. The relationship between red blood cell distribution width and the clinical outcomes in non-ST elevation myocardial infarction and unstable angina pectoris: a 3-year follow-up. Coron Artery Dis. 2012;23(5):330-336.

23. Ilhan E, Guvenc TS, Altay S, Cagdas M, Calik AN, Karaca $M$, Guzelburc $O$, et al. Predictive value of red cell distribution width in intrahospital mortality and postintervention thrombolysis in myocardial infarction flow in patients with acute anterior myocardial infarction. Coron Artery Dis. 2012;23(7):450-454

24. Isik T, Kurt M, Ayhan E, Tanboga IH, Ergelen M, Uyarel $H$. The impact of admission red cell distribution width on the development of poor myocardial perfusion after primary percutaneous intervention. Atherosclerosis. 2012;224(1):143-149.

25. Sun XP, Chen WM, Sun ZJ, Ding XS, Gao XY, Liang $\mathrm{SW}$, Zhao HQ, et al. Impact of red blood cell distribution width on long-term mortality in patients with ST-elevation myocardial infarction. Cardiology. 2014;128(4):343348.

26. Turcato G, Serafini V, Dilda A, Bovo C, Caruso B, Ricci G, Lippi G. Red blood cell distribution width independently predicts medium-term mortality and major adverse cardiac events after an acute coronary syndrome. Ann Transl Med. 2016;4(13):254.

27. Uyarel H, Ergelen M, Cicek G, Kaya MG, Ayhan E, Turkkan C, Yildirim E, et al. Red cell distribution width as a novel prognostic marker in patients undergoing primary angioplasty for acute myocardial infarction. Coron Artery Dis. 2011;22(3):138-144.

28. Wang YL, Hua Q, Bai CR, Tang Q. Relationship between red cell distribution width and short-term outcomes in acute coronary syndrome in a Chinese population. Intern Med. 2011;50(24):2941-2945.

29. Zorlu A, Yucel H, Kaya H, Korkmaz O, Yildirimli K, Bektasoglu G. Red cell distribution width (RDW) and increased risk of recurrent acute coronary syndrome. Acta Med Anatol. 2015;3(2):39-46.

30. Su C, Liao LZ, Song Y, Xu ZW, Mei WY. The role of red blood cell distribution width in mortality and cardiovascular risk among patients with coronary artery diseases: a systematic review and meta-analysis. J Thorac Dis. 2014;6(10):1429-1440.

31. Hansson GK. Inflammation, atherosclerosis, and coronary artery disease. N Engl J Med. 2005;352(16):16851695.

32. Allen LA, Felker GM, Mehra MR, Chiong JR, Dunlap $\mathrm{SH}$, Ghali JK, Lenihan DJ, et al. Validation and potential mechanisms of red cell distribution width as a prognostic 
marker in heart failure. J Card Fail. 2010;16(3):230-238.

33. Ycas JW, Horrow JC, Horne BD. Persistent increase in red cell size distribution width after acute diseases: A biomarker of hypoxemia? Clin Chim Acta. 2015;448:107-
117.

34. Patel HH, Patel HR, Higgins JM. Modulation of red blood cell population dynamics is a fundamental homeostatic response to disease. Am J Hematol. 2015;90(5):422-428. 